\title{
THE EFFECT OF WIND ON THE PROPAGATION OF AN IDEALIZED FOREST FIRE*
}

\author{
PETRO BABAK ${ }^{\dagger}$, ANNE BOURLIOUX $^{\ddagger}$, AND THOMAS HILLEN ${ }^{\dagger}$
}

\begin{abstract}
A reaction-diffusion model for the propagation of an idealized forest fire is revisited to include the effect of wind on the speed of propagation. We study the existence of one-dimensional travelling wave solutions. When the wind velocity is zero or when the wind blows from the burning region, the existence and uniqueness of the travelling wave is proved. In the case when the wind blows into the burning region, we show that there exist at least two travelling wave solutions for small wind speed, and there are no travelling wave solutions for large wind speed. The analysis relies on comparison principles whereby the questions of existence and uniqueness are addressed via the construction of appropriate lower and upper solutions for the travelling waves. The theoretical results are supplemented with numerical examples for each case of wind velocity. To show the nature of the travelling wave solutions, their stability is examined using numerical analysis.
\end{abstract}

Key words. combustion, travelling wave, wildland fire, adiabatic process, existence and nonexistence, uniqueness and nonuniqueness

AMS subject classifications. 92F05, 80A25, 60J70

DOI. $10.1137 / 080727166$

1. Introduction. In this paper we use continuum equations for wildland fire spread to study the effect of wind on the propagation speed of the fire front. A comprehensive understanding of the wind-flame interaction is one of the major challenges in the prediction of wildland fire propagation [11, 12, 16, 20, 21]. Current mathematical research on the interaction of fire and wind includes discrete computer models, cellular automata, front tracking models, and continuum models. There are several advantages and disadvantages of one model class over the other, and we believe that a comprehensive understanding can come only from a combined effort that considers all modelling approaches. In this paper we focus on a continuum model for the temperature distribution $T(t, x)$ and the fuel mass $Y(t, x)$. The model takes the form of a reaction-advection-diffusion system and is a generalization of classical reaction-diffusion models previously proposed for this application.

Three key assumptions in this work are (i) the wind is constant, (ii) there is no heat loss, and (iii) there is a critical ignition temperature $T_{i g}>0$ such that combustion takes place only where $T>T_{i g}$. Assumption (i) implies that the impact of the fire on the wind velocity field is neglected; this can be valid only if the fire intensity is fairly low. Assumption (ii) implies that, once a fire has started, it will never burn out. Although unrealistic, this assumption has little impact in the context of the current study. The fire front is driven by newly inflamed patches along the fire front, and the behavior of the fire in the burnt region has no significance on the forward spread. Assumption (ii) makes the analysis tractable. In future research we will try to relax

\footnotetext{
*Received by the editors June 13, 2008; accepted for publication (in revised form) August 17, 2009; published electronically November 4, 2009.

http://www.siam.org/journals/siap/70-4/72716.html

${ }^{\dagger}$ University of Alberta, Edmonton, AB, T6G 2G1, Canada (petro@ualberta.ca, thillen@ualberta. ca). The work of the first author was supported by MITACS and PIMS. The work of the second author was supported by NSERC and MITACS.

${ }^{\ddagger}$ Mathematiques et Statistique, Université de Montréal, Montreal, QC, H3C 3J7, Canada (anne. bourlioux@umontreal.ca). The work of this author was supported by NSERC and MITACS.
} 
this assumption and include heat loss. We expect, however, that the spread result will not change significantly.

We will show that the wind has an effect on the speed of an advancing fire front. To do so, we develop a new method of super- and subsolutions to prove the existence of travelling waves. If the wind is opposed to the spread of the fire (the so-called backward rate of spread), then we find two travelling waves, a fast wave and a slow one. We will discuss the significance of these waves later.

The paper is organized as follows. In subsection 1.1, we introduce the adiabatic continuum model, and we discuss the relevant literature. In section 2, we transform the problem into travelling wave coordinates, and we introduce meaningful boundary conditions. Furthermore, we introduce a transformation which is crucial for the remainder of the analysis. In section 3, we study travelling wave fronts in the case of no wind. This case has been studied in the literature (see $[5,6])$; however, we use an alternative method and introduce our new construction of super- and subsolutions. In section 4, we use this new construction to discuss the case including nonzero wind in detail. In section 5, we provide a case study analysis of travelling wave solutions for different types of reaction rates. In section 6 , we show our numerical investigations of the stability of the travelling wave solutions for different wind regimes. Finally, in section 7 we attempt to explain the nature of the combustion travelling waves and highlight some potentially interesting problems generated by our investigations. Throughout the paper, we show numerical solutions to illustrate the theoretical findings.

1.1. The model. We study a two-dimensional continuum model for the temperature $T(t, x)$ and fuel mass fraction $Y(t, x)$ which is based on a classical combustion model; see, e.g., [8]. The unit of the temperature $T$ is Kelvin and the fuel mass fraction (relative amount of fuel remaining) is noted as $Y \in[0,1]$. The evolution of $T$ is governed by an energy balance equation and the evolution of $Y$ accounts only for reaction $[7,19]$ :

$$
\begin{aligned}
& \rho C\left(\frac{\partial T}{\partial t}+\vec{w} \cdot \nabla T\right)=k \Delta T+\rho Q \cdot R(T,|w|) Y, \\
& \frac{\partial Y}{\partial t}=-R(T,|w|) Y
\end{aligned}
$$

where $\vec{w}\left(m s^{-1}\right)$ is the wind velocity (|w| denotes its speed), $\rho$ denotes the density of fuel $\left(\mathrm{kg} \cdot \mathrm{m}^{-3}\right), C$ is the specific heat of fuel $\left(J \cdot \mathrm{kg}^{-1} \cdot K^{-1}\right), k$ is the thermal conductivity of fuel $\left(J \cdot s^{-1} m^{-1} K^{-1}\right)$, and $Q$ is the heat (exothermicity) of combustion $\left(J \cdot k g^{-1}\right)$.

The fuel must be heated to the ignition temperature $T_{i g}$ before combustion starts. Therefore, the $T$-dependence of the reaction rate $R(T,|w|)$ is a function of ignition type; that is, if $T>T_{i g}$, then $R(T,|w|)>0$; otherwise $R(T,|w|)=0$. We will discuss in section 5.3 the possible dependence of the reaction rate on the wind speed $|w|$.

The results in this paper are obtained for general monotonically nondecreasing burning kinetics $R(T,|w|)$, where the wind velocity $|w|$ is assumed to be constant; hence we will mostly just write $R(T)$ in what follows. Our standard example is the Arrhenius law. It is based on the law of mass action and given by

$$
R(T,|w|)=A(|w|) e^{-\frac{E}{\hat{R} T}} \mathcal{H}\left(T-T_{i g}\right),
$$

where $\hat{R}$ is the universal gas constant (equal to $8.314 J \cdot \mathrm{mol}^{-1} \mathrm{~K}^{-1}$ ), $E$ is the activation energy $\left(J \cdot \mathrm{mol}^{-1}\right)$, and $\mathcal{H}(z)$ is the Heaviside step function. 
Similar systems have been studied for movable fuel (e.g., gases). The ratio of the diffusion coefficient of the temperature and of the fuel is called the Lewis number $L e$ [25]. A Lewis number of $L e=1$ would indicate that fuel and temperature have the same diffusivity. Our case of immobile fuel corresponds to the limit of $L e \rightarrow \infty$ because there is no spatial drift or diffusion term in the fuel equation for $Y$. Because of this asymmetry in the equations, the wind-convection term in system (1.1) cannot be removed using a standard translation of variables of the form $\xi=x-\vec{w} t$, as is the case for $L e=1$. In that case, the effect of advection in one dimension is trivial, unlike for the present situation.

Systems of the form (1.1) and generalizations thereof have been studied extensively in the literature. Most studies focus on numerical and experimental perspectives and also on asymptotic methods; see, e.g., [3, 9, 10, 13, 14, 15, 18, 22, 23, 25]. Directly related to our work is the work by Logak and Loubeau [5] and Logak [6], where the existence of travelling waves for the one-dimensional windless problem was shown using the Leray-Shauder topological degree theory. This case is revisited in section 3 with a new construction of super- and subsolutions. Our main result is the existence of travelling waves in the presence of nonzero wind. To the best of our knowledge, this case has not been studied previously.

2. Travelling waves. To study the travelling waves for system (1.1) we nondimensionalize the system and focus on a small number of necessary parameters. Let $L$ be the reference length scale. We define

$$
\tilde{x}=\frac{x}{L}, \quad \tilde{t}=\frac{k}{\rho C L^{2}} t, \quad \overrightarrow{\tilde{w}}=\frac{\rho C L}{k} \vec{w}, \quad \tilde{u}=\frac{C}{Q Y_{\infty}}\left(T-T_{\infty}\right), \quad \tilde{v}=\frac{Y}{Y_{\infty}},
$$

where $T_{\infty}$ denotes the ambient temperature and $Y_{\infty}$ the fuel supply in the unburned region.

Using these transformations and removing the tilde for convenience, system (1.1) becomes

$$
\begin{aligned}
& \frac{\partial u}{\partial t}+\vec{w} \cdot \nabla u=\Delta u+r(u,|w|) v, \\
& \frac{\partial v}{\partial t}=-r(u,|w|) v
\end{aligned}
$$

where

$$
r(u,|w|)=\frac{\rho C L^{2}}{k} R\left(u \frac{Q Y_{\infty}}{C}+T_{\infty}, \frac{k}{\rho C L}|w|\right) .
$$

The rescaled reaction rate $r(u)$ is also a function of ignition type. For the $u$-dependence of $r(u,|w|)$ and fixed $|w|$ we assume

$$
\left\{\begin{array}{l}
r(u,|w|)=0 \text { on }(-\infty, \theta), \\
r(.,|w|): \mathbb{R} \rightarrow \mathbb{R} \text { is a locally Lipschitz and nondecreasing function on }[\theta,+\infty), \\
\exists \underline{r}, \bar{r} \text { such that } 0<\underline{r}=r(\theta,|w|) \leq r(u,|w|) \leq \bar{r} \text { on }[\theta,+\infty),
\end{array}\right.
$$

where $\theta=\frac{C}{Q Y_{\infty}}\left(T_{i g}-T_{\infty}\right)$.

A travelling wave of (2.1) is a self-similar solution of the form $u\left(t, x_{1}, x_{2}\right)=$ $u(x-\vec{c} t)$ and $v\left(t, x_{1}, x_{2}\right)=v(x-\vec{c} t)$, where $\vec{c}$ is the velocity of the travelling wave 
front. For planar travelling waves we can assume that the wave advances in the $x_{1}$ direction, so we select $\eta=x_{1}-c t$, with $w=w_{1}$ and $c=c_{1}$, and we write the equations for the travelling waves as follows:

$$
\begin{aligned}
& (w-c) u^{\prime}=u^{\prime \prime}+r(u,|w|) v \\
& -c v^{\prime}=-r(u,|w|) v
\end{aligned}
$$

where $^{\prime}=d / d \eta$.

From now on we make two assumptions about the parameter values. We list them here now, although they will be motivated through the analysis later:

$$
\theta<1, \quad c>w .
$$

For boundary conditions we assume that ahead of the fire front the fuel mass fraction is $Y=Y_{\infty}$ and the temperature equals the ambient temperature $T=T_{\infty}$. In transformed coordinates this corresponds to

$$
u(+\infty)=0, \quad v(+\infty)=1 .
$$

Since we assume no heat loss, the fire will consume the fuel completely once started. Hence we assume

$$
v(-\infty)=0 .
$$

Under conditions (2.5) and (2.6) we can integrate system (2.3) and we find

$$
\begin{aligned}
& u^{\prime}=-c\left(\frac{c-w}{c} u+v-1\right), \\
& v^{\prime}=\frac{1}{c} r(u,|w|) v .
\end{aligned}
$$

Notice that imposing the boundary condition for $v$ at $\eta \rightarrow-\infty$ we can solve the $u$-equation in steady state. This gives the boundary condition for $u$ :

$$
u(-\infty)=\frac{c}{c-w},
$$

which is meaningful since we assumed $c>w$ in (2.4).

In order to define the travelling wave solution uniquely with respect to translations of the independent variable $\eta$, we fix the ignition point at $\eta=0$ in the moving coordinate system:

$$
u(0)=\theta .
$$

This splits the problem into two separate regions, the unburned region ahead of the wave, $\eta>0$, and the burning region $\eta \leq 0$.

2.1. Unburned region. Ahead of the fire front, the temperature satisfies $u<\theta$, and no fuel is consumed. Hence

$$
v(\eta)=1 \quad \text { for } \quad \eta>0
$$

In this case we can solve the equation for $u$ in (2.7) and obtain

$$
u(\eta)=\theta e^{-(c-w) \eta} \quad \text { for } \quad \eta>0 .
$$

Note that the boundary condition for $u$ given by (2.5) can be satisfied only if assumption (2.4) holds. 
2.2. Burning region. To be able to construct super- and subsolutions later, we introduce a variable transformation in the burning domain of $\eta \leq 0$. In that region $r(u)>0$ and we consider the problem for the left half-line:

$$
\begin{aligned}
u^{\prime} & =-c\left(\frac{c-w}{c} u+v-1\right), \\
v^{\prime} & =\frac{1}{c} r(u,|w|) v \\
u(-\infty) & =\frac{c}{c-w} \\
v(-\infty) & =0
\end{aligned}
$$

To generate homogeneous boundary conditions at $\eta=-\infty$, we introduce two new independent variables $U(\eta), V(\eta)$ as

$$
U(\eta):=1-\frac{c-w}{c} u(\eta), \quad V(\eta):=v(\eta)
$$

The inverse transformation for $U$ is given as

$$
u=\frac{c}{c-w}(1-U)
$$

Using this transformation we obtain from (2.12)

$$
\begin{aligned}
U^{\prime} & =(c-w)(V-U), \\
V^{\prime} & =\frac{1}{c} r\left(\frac{c}{c-w}(1-U),|w|\right) V \\
U(-\infty) & =0 \\
V(-\infty) & =0
\end{aligned}
$$

We study parts of the solution where $V$ can be written as a function of $U$. From (2.14) we obtain

$$
\frac{d V}{d U}=\frac{1}{c(c-w)} r\left(\frac{c}{c-w} 1-U,|w|\right) \frac{V}{V-U}, \quad V(0)=0
$$

Based on (2.15) we will construct our super- and subsolutions in the burning region.

3. Zero wind $(\boldsymbol{w}=\mathbf{0})$. We will first consider the case when $w=0$ and use $r(u, 0)=r(u)$. The travelling wave problem in this case can be written in the form

$$
\begin{array}{ll}
u^{\prime}=-c(u+v-1), \\
v^{\prime}=\frac{1}{c} r(u) v, \\
u(-\infty)=1, \quad u(+\infty)=0, \\
v(-\infty)=0, \quad v(+\infty)=1, \\
u(0)=\theta .
\end{array}
$$

The system of equations (3.1) has a continuum of stationary points $(u, v)=$ $(\alpha, 1-\alpha)$, with $\alpha \in(-\infty, \theta]$, and one isolated stationary point at $(u, v)=(1,0)$. The solution of problem (3.1) in the unburned region corresponding to $\eta>0$ can be directly taken from above (2.11), (2.10) and yields

$$
u(\eta)=\theta e^{-c \eta}, \quad v(\eta)=1, \quad \eta>0 .
$$


To analyze the behavior of the solution for $\eta<0$, we will use the transformation introduced earlier. In the case of $w=0(2.15)$ becomes

$$
\frac{d V}{d U}=\frac{1}{c^{2}} r(1-U) \frac{V}{V-U}, \quad V(0)=0 .
$$

The reaction term $r(1-U)$ on the right-hand side is nonincreasing in $U$; hence we use an upper and a lower bound of this term to find upper and lower solutions. For the reason that will be evident in the lemma about the monotonicity with respect to $c$ (Lemma 3.3), we first consider problem (3.2) on the interval $\left(0, U^{*}\right]$, where the value of $U^{*}$ belongs to $(0,1-\theta]$. In this case the reaction term $r(1-U)$ is bounded from below by $r\left(1-U^{*}\right)$ and from above by $r(1)$. For a lower solution $\underline{V}(U)$ and an upper solution $\bar{V}(U)$ on $\left(0, U^{*}\right]$ we propose the following initial value problems:

$$
\begin{array}{ll}
\frac{d \underline{V}}{d U}=\frac{1}{c^{2}} r\left(1-U^{*}\right) \frac{\underline{V}}{\underline{V}-U}, & \underline{V}(0)=0, \\
\frac{d \bar{V}}{d U}=\frac{1}{c^{2}} r(1) \frac{\bar{V}}{\bar{V}-U}, & \bar{V}(0)=0 .
\end{array}
$$

We study the properties of these solutions in detail.

Lemma 3.1 (boundedness and monotonicity). Let $V(U), \underline{V}(U)$, and $\bar{V}(U)$ be nontrivial solutions of the respective problems (3.2)-(3.4), and let $c>0$. Then $V(U)>$ $U, \underline{V}(U)>U$, and $\bar{V}(U)>U$ on $\left(0, U^{*}\right]$. Moreover, the functions $V(U), \underline{V}(U)$, and $\bar{V}(U)$ are strictly increasing on $\left(0, U^{*}\right]$.

Proof. Let us show that $V(U)>U$ on $\left(0, U^{*}\right]$. Using a contradiction argument, we assume that $V\left(U^{\sharp}\right)<U^{\sharp}$ for some $U^{\sharp}>0$. Then there are three possible cases for the value $V\left(U^{\sharp}\right): V\left(U^{\sharp}\right)=0, V\left(U^{\sharp}\right)<0$, and $V\left(U^{\sharp}\right)>0$. The condition $V\left(U^{\sharp}\right)=0$ implies that $V(U)$ is a trivial solution of (3.2). In the case $V\left(U^{\sharp}\right)<0$, the function $V(U)$ is nondecreasing on $\left(0, U^{\sharp}\right]$; therefore, $V(0) \leq V\left(U^{\sharp}\right)<0$, which contradicts the initial condition in (3.2). In the last case $V\left(U^{\sharp}\right)>0$, note that $d V / d U<0$ if $U>V(U)>0$. Therefore, the trajectory of the function $V(U)$ has to cross the line $V=U$ in order to connect the initial point $(0,0)$ with the point $\left(U^{\sharp}, V\left(U^{\sharp}\right)\right)$. Let $V\left(U^{b}\right)=U^{b}$ for some $U^{b} \in\left(0, U^{\sharp}\right)$. Then $\lim _{U \rightarrow U^{b}-0} d V / d U(U) \leq 1$ in order to pass from the domain $V>U$ to the domain $V<U$ at the point $U^{b}$. However, this contradicts problem (3.2), since $\lim _{V \rightarrow U^{b}-0} d V / d U=+\infty$.

Finally, the statement regarding the lower and upper solutions can be directly verified using the explicit form of the nontrivial solutions for problems (3.3) and (3.4):

$$
\underline{V}=\left(1+\frac{1}{c^{2}} r\left(1-U^{*}\right)\right) U, \quad \bar{V}=\left(1+\frac{1}{c^{2}} r(1)\right) U .
$$

LEMma 3.2 (upper and lower solutions). Let $V(U), \underline{V}(U)$, and $\bar{V}(U)$ be nontrivial solutions of problems (3.2)-(3.4), and let $c>0$. Then $\underline{V}(U)<V(U)$ on $\left(0, U^{*}\right)$, and $V(U)<\bar{V}(U)$ on $\left(0, U^{*}\right]$.

Proof. Using a proof by contradiction we will show that $\underline{V}(U)<V(U)$ on $\left(0, U^{*}\right)$. There are two possible cases. In the first case, we assume that $\underline{V}(U)>V(U)$ on $\left(0, U^{b}\right)$ for some $0<U^{b} \leq U^{*}$; then, in view of Lemma 3.1, it follows that

$$
\begin{aligned}
0 & \leq \int_{0}^{U^{b}} \frac{d(\underline{V}-V)}{d U} d U=\frac{1}{c^{2}} \int_{0}^{U^{b}}\left[r\left(1-U^{*}\right) \frac{\underline{V}}{\underline{V}-U}-r(1-U) \frac{V}{V-U}\right] d U \\
& \leq \frac{1}{c^{2}} r\left(1-U^{*}\right) \int_{0}^{U^{b}}\left[\frac{\underline{V}}{\underline{V}-U}-\frac{V}{V-U}\right] d U
\end{aligned}
$$




$$
=-\frac{1}{c^{2}} r\left(1-U^{*}\right) \int_{0}^{U^{b}} \frac{U(\underline{V}-V)}{(\underline{V}-U)(V-U)} d U<0 .
$$

In the second case, let $\underline{V}\left(U^{\sharp}\right)=V\left(U^{\sharp}\right)$ for some $U^{\sharp} \in\left(0, U^{*}\right)$, and let $\underline{V}(U)<$ $V(U)$ on $\left(0, U^{\sharp}\right)$. Then the function $(\underline{V}-V)(U)$ attains its local maximum on the interval $\left(0, U^{*}\right]$ in the point $U^{\sharp}$. Thus $d(\underline{V}-V) / d U\left(U^{\sharp}\right) \geq 0$. However, (3.2) and (3.3) imply that $d(\underline{V}-V) / d U\left(U^{\sharp}\right)<0$ for any $U^{\sharp} \in\left(0, U^{*}\right)$. Finally, using analogous arguments we can prove that $V(U)<\bar{V}(U)$ for $U \in\left(0, U^{*}\right)$.

LEMMA 3.3 (monotonicity in the wave speed $c$ ). Let $V_{c}(U)$ be a nontrivial solution of problem (3.2) for $c>0$. If $0<c_{1}<c_{2}$, then $V_{c_{1}}(U)>V_{c_{2}}(U)$ for $U \in(0,1-\theta]$.

Proof. To prove this by contradiction, we assume first that there exists a $U^{\sharp} \in$ $(0,1-\theta]$ such that $V_{c_{1}}\left(U^{\sharp}\right)=V_{c_{2}}\left(U^{\sharp}\right)$ and $V_{c_{1}}(U)>V_{c_{2}}(U)$ on $\left(0, U^{\sharp}\right)$. Then the function $V_{c_{1}}(U)-V_{c_{2}}(U)$ attains its local minimum on the interval $\left(0, U^{\sharp}\right]$ in the point $U^{\sharp}$; therefore, $d\left(V_{c_{1}}(U)-V_{c_{2}}(U)\right) / d U\left(U^{\sharp}\right) \leq 0$. However, from problem (3.2) we infer that $d\left(V_{c_{1}}(U)-V_{c_{2}}(U)\right) / d U\left(U^{\sharp}\right)>0$.

Let us now assume that $V_{c_{1}}(U)<V_{c_{2}}(U)$ on $\left(0, U^{b}\right]$. Since the function $r(u)$ is nondecreasing and continuous, there exists a value $U^{* *} \in\left(0, U^{\mathrm{b}}\right]$ such that

$$
r\left(1-U^{* *}\right) \geq \frac{c_{1}^{2}}{c_{2}^{2}} r(1) .
$$

But in this case for any $U \in\left(0, U^{* *}\right]$, in view of Lemmas 3.1 and 3.2, and equations (3.5),

$$
0>V_{c_{1}}(U)-V_{c_{2}}(U)>\underline{V}_{c_{1}}(U)-\bar{V}_{c_{2}}(U)=\left(\frac{1}{c_{1}^{2}} r\left(1-U^{*}\right)-\frac{1}{c_{2}^{2}} r(1)\right) U \geq 0,
$$

where the lower and upper solutions are defined from problems (3.3) and (3.4) for $U^{*}=U^{* *}$.

Given these three solutions $\underline{V}(U), V(U)$, and $\bar{V}(U)$ for $U^{*}=1-\theta$, we will now use the equation for $u$ to define the corresponding super- and subsolutions $(\underline{u}, \underline{v})$ and $(\bar{u}, \bar{u})$. We now assume that $u(\eta), \underline{u}(\eta)$, and $\bar{u}(\eta)$ on $(-\infty, 0)$ satisfy

$$
\begin{array}{lll}
u^{\prime}=-c(u+V(U)-1), & u(-\infty)=1, & u(0)=\theta, \\
\underline{u}^{\prime}=-c(\underline{u}+\underline{V}(\underline{U})-1), & \underline{u}(-\infty)=1, & \underline{u}(0)=\theta, \\
\bar{u}^{\prime}=-c(\bar{u}+\bar{V}(\bar{U})-1), & \bar{u}(-\infty)=1, & \bar{u}(0)=\theta,
\end{array}
$$

where $V(U), \underline{V}(U)$, and $\bar{V}(U)$ are nontrivial solutions of (3.2), (3.3), and (3.4) for $U^{*}=1-\theta$. Using the notation introduced in (2.5) for the present case of $w=0$, we have $U(\eta)=1-u(\eta)$, and $v(\eta)=V(U(\eta))=V(1-u(\eta))$, with similar expressions for $\underline{v}(\eta)=\underline{V}(1-\underline{u}(\eta))$ and $\bar{v}(\eta)=\bar{V}(1-\bar{u}(\eta))$. Then the vector functions $(u(\eta), v(\eta))$, $(\underline{u}(\eta), \underline{v}(\eta))$, and $(\bar{u}(\eta), \bar{v}(\eta))$ satisfy, respectively,

$$
\begin{aligned}
& \left\{\begin{array}{l}
u^{\prime}=-c(u+v-1), \quad v^{\prime}=\frac{1}{c} \operatorname{vr}(u), \\
u(-\infty)=1, \quad v(-\infty)=0, \quad u(0)=\theta,
\end{array}\right. \\
& \left\{\begin{array}{l}
\underline{u}^{\prime}=-c(\underline{u}+\underline{v}-1), \quad \underline{v}^{\prime}=\frac{1}{c} \underline{v} r(\theta), \\
\underline{u}(-\infty)=1, \quad \underline{v}(-\infty)=0, \quad \underline{u}(0)=\theta,
\end{array}\right. \\
& \left\{\begin{array}{l}
\bar{u}^{\prime}=-c(\bar{u}+\bar{v}-1), \quad \bar{v}^{\prime}=\frac{1}{c} \bar{v} r(1), \\
\bar{u}(-\infty)=1, \quad \bar{v}(-\infty)=0, \quad \bar{u}(0)=\theta .
\end{array}\right.
\end{aligned}
$$


The solutions of problems (3.7) and (3.8) can be explicitly obtained and are given as

$$
\begin{aligned}
& \underline{u}(\eta)=1-(1-\theta) \exp \left(\frac{1}{c} r(\theta) \eta\right), \\
& \underline{v}(\eta)=(1-\theta)\left(1+\frac{1}{c^{2}} r(\theta)\right) \exp \left(\frac{1}{c} r(\theta) \eta\right)=(1-\underline{u}(\eta))\left(1+\frac{1}{c^{2}} r(\theta)\right), \\
& \bar{u}(\eta)=1-(1-\theta) \exp \left(\frac{1}{c} r(1) \eta\right), \\
& \bar{v}(\eta)=(1-\theta)\left(1+\frac{1}{c^{2}} r(1)\right) \exp \left(\frac{1}{c} r(1) \eta\right)=(1-\bar{u}(\eta))\left(1+\frac{1}{c^{2}} r(1)\right) .
\end{aligned}
$$

Next we analyze the phase portrait of system (3.6). The point $(1,0)$ is a saddle point for $c>0$. The unstable eigenvalue of the linearization of $(3.6)$ at $(1,0)$ is $\lambda_{1}=\frac{1}{c} r(1)$, and the corresponding eigenvector is given by $\phi_{1}=\left(-1 /\left(1+\frac{1}{c^{2}} r(1)\right), 1\right)$. In view of the stable manifold theorem, there exists a unique solution of problem (3.6) which starts (for $\eta=-\infty$ ) at the point $(1,0)$ and which is tangential to $\phi_{1}$ at $(1,0)$. The values of the components of $\phi_{1}$ and the property that $V(U)>U$ from Lemma 3.1 imply that this solution is in the domain $\{(u, v): u<1, v+u>1\}$.

Furthermore, from Lemmas 3.1-3.3 we can infer the following estimates.

Lemma 3.4. Let $(u, v)$ be a solution of problem (3.6) for $c>0$, and let $(\underline{u}, \underline{v})$ and $(\bar{u}, \bar{v})$ be a pair of the lower and upper solutions of (3.7) and (3.8) for $(u, v)$. Then for $\eta \in(-\infty, 0]$

- $\theta \leq u(\eta)<1$ and $u(\eta)+v(\eta)>1$,

- $\underline{v}(0)<v(0)<\bar{v}(0)$, and the trajectory of $(u(\eta), v(\eta))$ lies between two trajectories $(\underline{u}(\eta), \underline{v}(\eta))$ and $(\bar{u}(\eta), \bar{v}(\eta))$ for $\eta<0$,

- $v(0)$ is a strictly decreasing function of $c, c>0$, and

- $-c \bar{v}(0) \leq-c v(0) \leq u^{\prime}(\eta)<0$ and $0<v^{\prime}(\eta) \leq c r(1) v(0) \leq c r(1) \bar{v}(0)$.

Figure 3.1 presents an illustration for Lemma 3.4. Specifically, the upper and lower solutions to problem (3.6) are shown for two different positive values of $c$. The solution of problem (3.6) for $\eta<0$ is located between corresponding lower and upper solutions. Figure 3.1 also shows that the value of $v(0)$ varies with respect to $c$. Next we show that there is exactly one value for $c>0$ such that $v(0)=1$. This solution corresponds to a travelling wave connection.

TheOREM 3.5 (unique wave speed for $w=0$ ). There exists a unique $c>0$ such that the solution of problem (3.6) satisfies the condition $v(0)=1$. Moreover,

$$
0<\underline{c}<c<\bar{c}
$$

where $\underline{c}=\sqrt{\frac{1-\theta}{\theta} r(\theta)}$ and $\bar{c}=\sqrt{\frac{1-\theta}{\theta} r(1)}$.

Proof. Since the functions $v(0), \underline{v}(0)$, and $\bar{v}(0)$ are strictly decreasing with respect to $c, c>0$, and

$$
\lim _{c \rightarrow+\infty} \underline{v}(0)=\lim _{c \rightarrow+\infty} \bar{v}(0)=1-\theta, \quad \lim _{c \rightarrow+0} \underline{v}(0)=\lim _{c \rightarrow+0} \bar{v}(0)=+\infty,
$$

there exists a unique $c>0$ such that $v(0)=1$. Clearly, in this case $\underline{v}(0)<1<\bar{v}(0)$, and, therefore, $c$ satisfies inequality (3.9), where $\underline{c}$ is the solution of the equation $\underline{v}(0)=1$, and $\bar{c}$ is the solution of the equation $\bar{v}(0)=1$. See also Figure 3.2 for illustrations of this proof. 


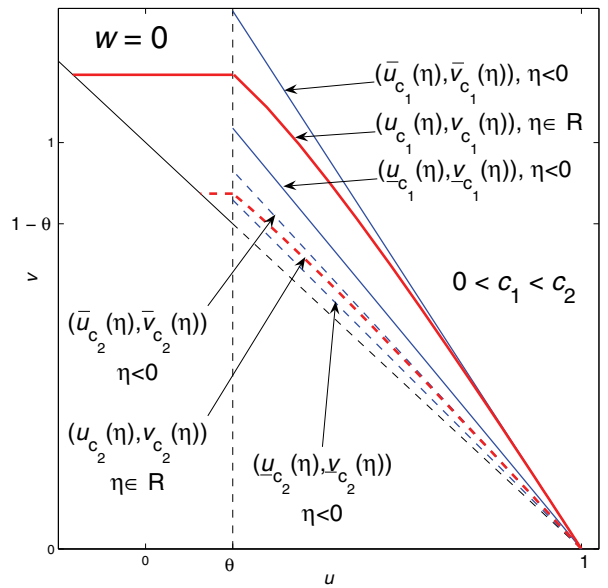

FIG. 3.1. Case $w=0$. Schematic phase plane of the $u$, v-system for two different positive values of $c$. The heteroclinic orbits $\left(u_{c_{1}}(\eta), v_{c_{1}}(\eta)\right)$ and $\left(u_{c_{2}}(\eta), v_{c_{2}}(\eta)\right), \eta \in \mathbb{R}$, connect the saddle stationary point $(0,1)$ with the set of stationary points $\{(u, v)=(\tilde{u}, 1-\tilde{u}): \tilde{u} \in(-\infty, \theta]\}$. The values $\left(u_{c}(\infty), v_{c}(\infty)\right)$ that belong to the set of stationary points and $\left(u_{c}(0), v_{c}(0)\right)$ vary monotonically with respect to $c$. The upper and lower solutions are shown for each heteroclinic orbit when $\eta<0$.

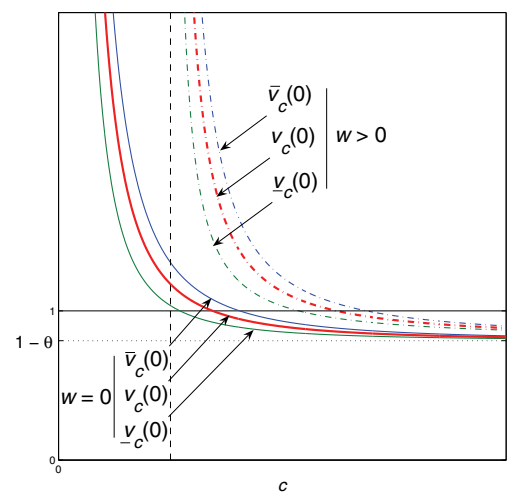

Fig. 3.2. Values of $v_{c}(0), \underline{v}_{c}(0)$, and $\bar{v}_{c}(0)$ as a function of wave speed $c$. We show both cases, $w=0$ and $w>0$.

Summarizing the behavior of the solutions for positive and negative $\eta$, we can formulate the following existence and uniqueness theorem.

THEOREM 3.6. If $w=0$, then system (2.1) has a unique travelling front solution governed by equations (3.1). The speed $c$ of the travelling front satisfies inequality (3.9). The trajectory $(u(\eta), v(\eta)), \eta \in \mathbb{R}$, of the travelling front belongs to the domain

$$
\{(u, v): v+u \geq 1,0 \leq v \leq 1,0 \leq u \leq 1\} .
$$

Moreover,

$$
\begin{aligned}
0 & \leq v^{\prime}(\eta) \leq r(1) \sqrt{\frac{\theta}{(1-\theta) r(1 / \theta)}}, \\
-\sqrt{\frac{1-\theta}{\theta} r(1)} & \leq u^{\prime}(\eta)<0, \quad-r(1) \leq u^{\prime \prime}(\eta) \leq \frac{1-\theta}{\theta} r(1) .
\end{aligned}
$$




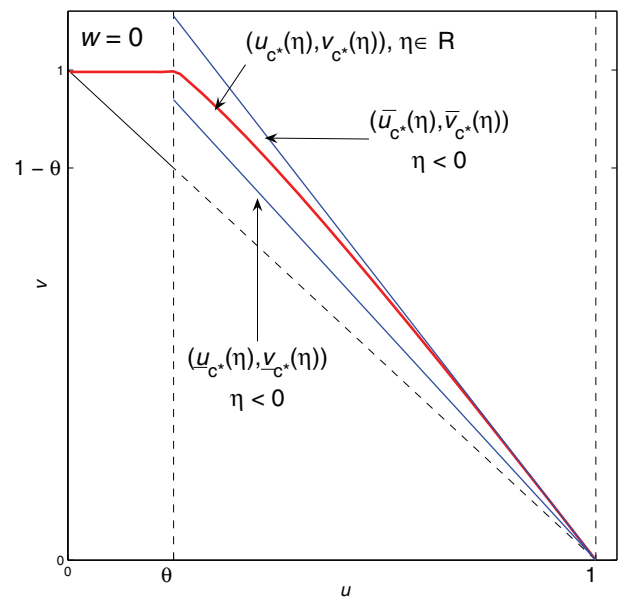

(a)

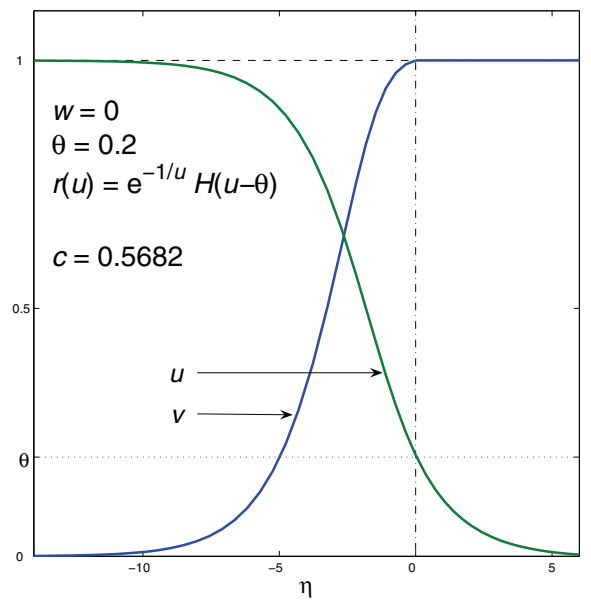

(b)

FIG. 3.3. Case $w=0$. (a) Schematic phase plane of the $u$, $v$-system for the solution of problem (3.1). The heteroclinic orbit $\left(u_{c^{*}}(\eta), v_{c^{*}}(\eta)\right), \eta \in \mathbb{R}$, connects the saddle stationary point $(1,0)$ with the point $(0,1)$ of the set of stationary points. The upper and lower solutions are shown for the solution when $\eta<0$. (b) Numerical approximation to the travelling wave solution given by equations (3.1).

The construction underlying Theorem 3.6 is illustrated in Figure 3.3(a). The estimates for the derivatives follow directly from the underlying differential equations. In Figure 3.3(b) we show a numerical approximation of the travelling front for $\theta=$ 0.2 and $r(u)=e^{-1 / u} \mathcal{H}(u-\theta)$. In this case the speed of the travelling front is approximately $c=0.5682$.

4. Nonzero wind. The travelling wave problem in the case of $w \neq 0$ can be written in the form

$$
\begin{aligned}
& u^{\prime}=-c\left(\frac{c-w}{c} u+v-1\right), \\
& v^{\prime}=\frac{1}{c} r(u,|w|) v, \\
& u(-\infty)=\frac{c}{c-w}, \quad u(+\infty)=0, \\
& v(-\infty)=0, \quad v(+\infty)=1, \\
& u(0)=\theta .
\end{aligned}
$$

For each fixed $c$ and $w$ the system (4.1) has a continuum of steady states of the form $(u, v)=\left(\alpha, 1-\frac{c-w}{c} \alpha\right)$, where $\alpha \in(-\infty, \theta]$, and one isolated stationary point $(u, v)=\left(\frac{c}{c-w}, 0\right)$. Clearly, the condition

$$
\frac{c}{c-w}>\theta
$$

should be satisfied; otherwise $u(-\infty) \leq \theta$, and there is no travelling front connecting $\left(0, \frac{c}{c-w}\right)$ and $(1,0)$.

4.1. Case $\boldsymbol{w}>\mathbf{0}$. Here we fix the wind $w>0$ and again write $r(u)$ instead of $r(u,|w|)$. It follows from the second condition in (2.4) that condition (4.2) is automatically satisfied. 
For each $c>w$, the steady state $\left(\frac{c}{c-w}, 0\right)$ is a saddle point. The positive eigenvalue of the linearization is $\lambda_{1}=\frac{1}{c} r\left(\frac{c}{c-w}\right)$ and the corresponding eigenvector is $\phi_{1}=$ $\left(-\left[\frac{c-w}{c}+\frac{1}{c^{2}} r\left(\frac{c}{c-w}\right)\right]^{-1}, 1\right)$. Clearly, this vector lies between the vectors $\left(-\frac{c}{c-w}, 1\right)$ and $(0,1)$. Therefore, in view of the stable manifold theorem, there exists a unique solution of problem (4.1) which starts for $\eta=-\infty$ at this steady state and which is tangential to $\phi_{1}$ at $\left(\frac{c}{c-w}, 0\right)$. Moreover, we show in Lemma 4.3 that the solution lies in the domain $\left\{(u, v): v+\frac{c-w}{c} u>1, u<\frac{c}{c-w}\right\}$.

Let us consider trajectories of $(4.1)$ in the burning region $(\eta<0)$ for two different values of front speed $c_{1}, c_{2}$, with $w<c_{1}<c_{2}$. These trajectories do not intersect each other. Indeed, if we assume they intersect at $\left(u_{c_{1}}\left(\eta_{1}\right), v_{c_{1}}\left(\eta_{1}\right)\right)=\left(u_{c_{2}}\left(\eta_{2}\right), v_{c_{2}}\left(\eta_{2}\right)\right)$, then we have from (4.1) that

$$
\frac{v_{c_{2}}^{\prime}\left(\eta_{2}\right)}{v_{c_{1}}^{\prime}\left(\eta_{1}\right)}=\frac{c_{1}}{c_{2}}<1
$$

However, again from (4.1) we have

$$
v_{c_{1}}^{\prime}\left(\eta_{1}\right)-v_{c_{2}}^{\prime}\left(\eta_{2}\right)=\frac{\left(c_{2}-c_{1}\right)}{c_{1} c_{2}} r\left(u_{c_{1}}\left(\eta_{1}\right)\right) v_{c_{1}}\left(\eta_{1}\right)>0,
$$

which contradicts the earlier statement. Hence there are no intersections. This automatically implies that $v_{c_{1}}(0)>v_{c_{2}}(0)$ when $w<c_{1}<c_{2}$.

Now, similar to the case with $w=0$, we introduce auxiliary problems for the functions $V(U), \underline{V}(U)$, and $\bar{V}(U)$ to construct super- and subsolutions. We fix $U^{*}=$ $1-\theta \frac{c-w}{c}$ and study for $U \in\left[0, U^{*}\right]$ the following initial value problems:

$$
\begin{array}{ll}
\frac{d V}{d U}=\frac{1}{c(c-w)} r\left(\frac{c}{c-w} 1-U\right) \frac{V}{V-U}, & V(0)=0, \\
\frac{d \underline{V}}{d U}=\frac{1}{c(c-w)} r(\theta) \frac{\underline{V}}{V-U}, & \underline{V}(0)=0, \\
\frac{d \bar{V}}{d U}=\frac{1}{c(c-w)} r\left(\frac{c}{c-w}\right) \frac{\bar{V}}{\bar{V}-U}, & \bar{V}(0)=0 .
\end{array}
$$

By analogy to the case $w=0$ we can prove the following lemmas.

LEMma 4.1 (boundedness and monotonicity). Let $V(U), \underline{V}(U)$, and $\bar{V}(U)$ be nontrivial solutions of problems (4.3)-(4.5), and let $c>0$. Then $\underline{V}(U)>U, V(U)>$ $U$, and $\bar{V}(U)>U$ on $\left(0, U^{*}\right]$. Moreover, the functions $V(U), \underline{V}(U)$, and $\bar{V}(U)$ are strictly increasing on $\left(0, U^{*}\right]$.

LEMMA 4.2 (upper and lower solutions). Let $V(U), \underline{V}(U)$, and $\bar{V}(U)$ be nontrivial solutions of problems (4.3)-(4.5), and let $c>0$. Then $\underline{V}(U)<V(U)$ on $U \in\left(0, U^{*}\right)$, and $V(U)<\bar{V}(U)$ on $U \in\left(0, U^{*}\right]$.

We define the functions $u(\eta), \underline{u}(\eta)$, and $\bar{u}(\eta)$ on $(-\infty, 0)$ as follows:

$$
\begin{array}{lll}
u^{\prime}=-c\left(\frac{c-w}{c} u+V\left(1-\frac{c-w}{c} u\right)-1\right), & u(-\infty)=\frac{c}{c-w}, \quad u(0)=\theta, \\
\underline{u}^{\prime}=-c\left(\frac{c-w}{c} \underline{u}+\underline{V}\left(1-\frac{c-w}{c} \underline{u}\right)-1\right), & \underline{u}(-\infty)=\frac{c}{c-w}, \quad \underline{u}(0)=\theta, \\
\bar{u}^{\prime}=-c\left(\frac{c-w}{c} \bar{u}+\bar{V}\left(1-\frac{c-w}{c} \bar{u}\right)-1\right), & \bar{u}(-\infty)=\frac{c}{c-w}, \quad \bar{u}(0)=\theta,
\end{array}
$$


where $V(U), \underline{V}(U)$, and $\bar{V}(U)$ are nontrivial solutions of (4.3), (4.4), and (4.5).

Then the vector functions $(u(\eta), v(\eta)),(\underline{u}(\eta), \underline{v}(\eta))$, and $(\bar{u}(\eta), \bar{v}(\eta))$ with $v(\eta)=$ $V\left(1-\frac{c-w}{c} u(\eta)\right), \underline{v}(\eta)=\underline{V}\left(1-\frac{c-w}{c} \underline{u}(\eta)\right)$, and $\bar{v}(\eta)=\bar{V}\left(1-\frac{c-w}{c} \bar{u}(\eta)\right)$ satisfy the problems

$$
\begin{aligned}
& \left\{\begin{array}{l}
u^{\prime}=-c\left(\frac{c-w}{c} u+v-1\right), \quad v^{\prime}=\frac{1}{c} r(u) v, \\
u(-\infty)=\frac{c}{c-w}, \quad v(-\infty)=0, \quad u(0)=\theta,
\end{array}\right. \\
& \left\{\begin{array}{l}
\underline{u}^{\prime}=-c\left(\frac{c-w}{c} \underline{u}+\underline{v}-1\right), \quad \underline{v}^{\prime}=\frac{1}{c} r(\theta) \underline{v}, \\
\underline{u}(-\infty)=\frac{c}{c-w}, \quad \underline{v}(-\infty)=0, \quad \underline{u}(0)=\theta,
\end{array}\right. \\
& \left\{\begin{array}{l}
\bar{u}^{\prime}=-c\left(\frac{c-w}{c} \bar{u}+\bar{v}-1\right), \quad \bar{v}^{\prime}=\frac{1}{c} r\left(\frac{c}{c-w}\right) \bar{v}, \\
\bar{u}(-\infty)=\frac{c}{c-w}, \quad \bar{v}(-\infty)=0, \quad \bar{u}(0)=\theta .
\end{array}\right.
\end{aligned}
$$

The solutions of problems (4.7) and (4.8) are

$$
\begin{aligned}
& \left\{\begin{aligned}
\underline{u}(\eta) & =\frac{c}{c-w}-\frac{c}{c-w}\left(1-\frac{c-w}{c} \theta\right) \exp \left(\frac{1}{c} r(\theta) \eta\right), \\
\underline{v}(\eta) & =\left(1-\frac{c-w}{c} \theta\right)\left(1+\frac{1}{c(c-w)} r(\theta)\right) \exp \left(\frac{1}{c} r(\theta) \eta\right) \\
& =\left(1+\frac{1}{c(c-w)} r(\theta)\right)\left(1-\frac{c-w}{c} \underline{u}(\eta)\right),
\end{aligned}\right. \\
& \left\{\begin{aligned}
\bar{u}(\eta) & =\frac{c}{c-w}-\frac{c}{c-w}\left(1-\frac{c-w}{c} \theta\right) \exp \left(\frac{1}{c} r\left(\frac{c}{c-w}\right) \eta\right), \\
\bar{v}(\eta) & =\left(1-\frac{c-w}{c} \theta\right)\left(1+\frac{1}{c(c-w)} r\left(\frac{c}{c-w}\right)\right) \exp \left(\frac{1}{c} r\left(\frac{c}{c-w}\right) \eta\right) \\
& =\left(1+\frac{1}{c(c-w)} r\left(\frac{c}{c-w}\right)\right)\left(1-\frac{c-w}{c} \bar{u}(\eta)\right) .
\end{aligned}\right.
\end{aligned}
$$

From Lemmas 4.1 and 4.2 we can infer the following estimates.

Lemma 4.3. Let $(u, v)$ be a solution of problem (4.6) for $c>w>0$, and let $(\underline{u}, \underline{v})$ and $(\bar{u}, \bar{v})$ be a pair of the lower and upper solutions of problems (4.7) and (4.8) for $(u, v)$. Then for $\eta \in(-\infty, 0]$

- $\theta \leq u(\eta)<\frac{c}{c-w}$ and $\frac{c-w}{c} u(\eta)+v(\eta)>1$,

- $\underline{v}(0)<v(0)<\bar{v}(0)$, and the trajectory of $(u(\eta), v(\eta))$ lies between two trajectories $(\underline{u}(\eta), \underline{v}(\eta))$ and $(\bar{u}(\eta), \bar{v}(\eta))$ for $\eta<0$,

- $v(0)$ is a strictly decreasing function of $c, c>0$, and

- $-c \bar{v}(0) \leq-c v(0) \leq u^{\prime}(\eta)<0$ and $0<v^{\prime}(\eta) \leq \operatorname{cr}\left(\frac{c}{c-w}\right) v(0) \leq c r\left(\frac{c}{c-w}\right) \bar{v}(0)$.

Figure 4.1 shows an illustration for Lemma 4.3. The heteroclinic orbits for different values of $c$ start from different saddle points for $\eta=-\infty$. The solution of problem (4.6) for $\eta<0$ is located between corresponding lower and upper solutions. Figure 4.1 also shows that the value of $v(0)$ monotonically varies with respect to $c$.

THEOREM 4.4. If $w>0$, then there exists a unique $c$ such that the solution of problem (4.6) satisfies the conditions $v(0)=1$ and $u(0)=\theta$. Moreover,

$$
0<w<\underline{c}<c<\bar{c}
$$




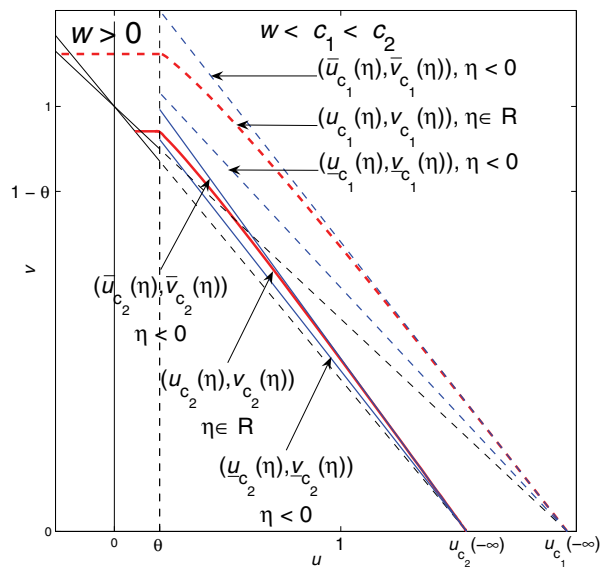

FIG. 4.1. Case $w>0$. Schematic phase plane of the $u, v$-system for two different positive values of $c$. The heteroclinic orbits $\left(u_{c_{1}}(\eta), v_{c_{1}}(\eta)\right)$ and $\left(u_{c_{2}}(\eta), v_{c_{2}}(\eta)\right), \eta \in \mathbb{R}$, connect the corresponding saddle stationary points distinct from $(1,0)$ with the corresponding set of stationary points. The values $\left(u_{c}(0), v_{c}(0)\right)$ vary monotonically with respect to $c$. The upper and lower solutions are shown for each heteroclinic orbit when $\eta<0$.

where $\underline{c}$ and $\bar{c}$ are unique solutions of the respective equations $\underline{v}(0)=1$ and $\bar{v}(0)=1$ that exceed $w$.

Proof. Since the functions $v(0), \underline{v}(0)$, and $\bar{v}(0)$ are strictly decreasing with respect to $c>0$, and

$$
\lim _{c \rightarrow+\infty} \underline{v}(0)=\lim _{c \rightarrow+\infty} \bar{v}(0)=1-\theta, \quad \lim _{c \rightarrow w+} \underline{v}(0)=\lim _{c \rightarrow w+} \bar{v}(0)=+\infty,
$$

there exists a unique $c>0$ that $v(0)=1$. Clearly, in this case $\underline{v}(0)<1<\bar{v}(0)$, and, therefore, $c$ satisfies inequality (4.11). The upper and lower estimates for the speed, $\underline{c}, \bar{c}$, are given implicitly through the conditions $\underline{v}(0)=1$ and $\bar{v}(0)=1$. See also Figure 3.2 for illustrations of this proof.

Summarizing the behavior of the solutions for positive and negative $\eta$, we can formulate the following existence and uniqueness theorem.

THEOREM 4.5. If $w>0$, then system (2.1) has a unique travelling front solution governed by equations (4.1). The speed $c$ of the travelling front satisfies inequality (4.11). The trajectory $(u(\eta), v(\eta)), \eta \in \mathbb{R}$, of the travelling front belongs to the domain

$$
\left\{(U, V): V+\bar{c}-w \bar{c} U \geq 1,0 \leq V \leq 1,0 \leq U \leq \frac{\underline{c}}{\underline{c}-w}\right\} .
$$

Moreover,

$$
\begin{gathered}
0 \leq v^{\prime}(\eta) \leq \frac{1}{\underline{c}} r\left(\frac{\underline{c}}{\underline{c}-w}\right), \\
-\bar{c} \leq u^{\prime}(\eta)<0, \quad-r\left(\frac{\underline{c}}{\underline{c}-w}\right) \leq u^{\prime \prime}(\eta) \leq \bar{c}(\bar{c}-w) .
\end{gathered}
$$

An illustration for the statements of this theorem is given in Figure 4.2(a). A numerical approximation of the travelling front is shown in Figure 4.2(b) for $w=0.2$ and $\theta=0.2$. In this case the speed of the travelling front is approximately $c=$ $0.8370>w$. 


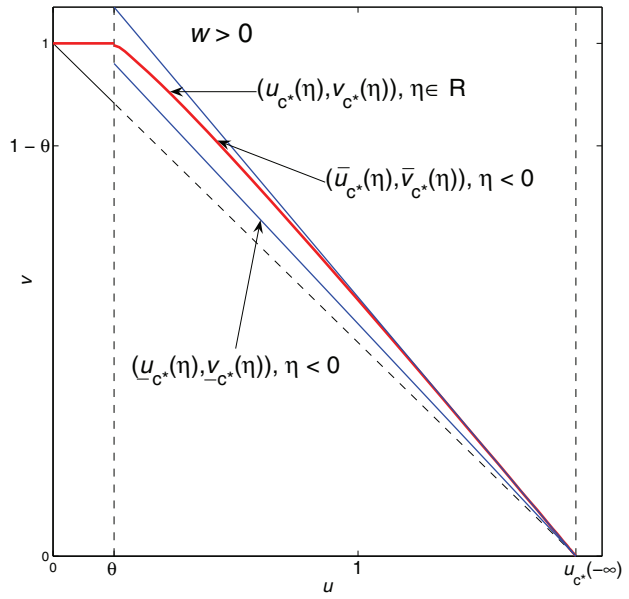

(a)

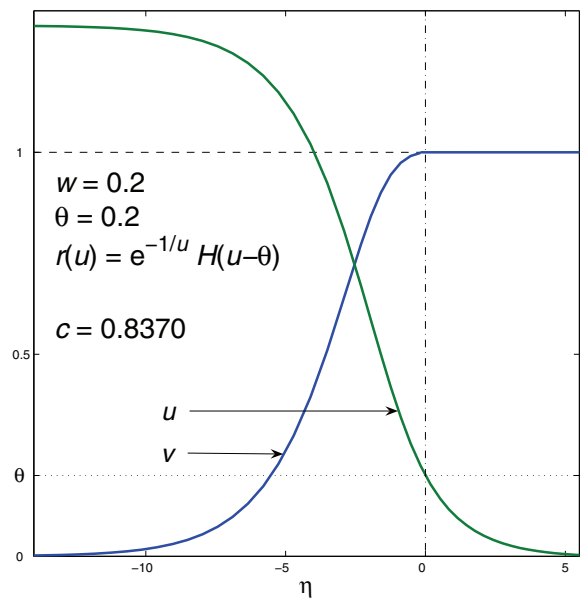

(b)

Fig. 4.2. Case $w>0$. (a) Schematic phase plane of the $u, v$-system for the solution of problem (4.1) with $w>0$. The heteroclinic orbit $\left(u_{c^{*}}(\eta), v_{c^{*}}(\eta)\right), \eta \in \mathbb{R}$, connects the saddle stationary point $\left(c^{*} /\left(c^{*}-w\right), 0\right)$ with the the point $(0,1)$ of the corresponding set of stationary points. The upper and lower solutions are shown for the solution when $\eta<0$. (b) Numerical approximation to the travelling wave problem given by equations (4.1) for $w>0$.

4.2. Case $\boldsymbol{w}<0$. Notice that to have a meaningful nontrivial steady state we need condition (4.2). Formulated as a condition for $c$ (4.2) becomes

$$
c>-w \frac{\theta}{1-\theta}=: c_{0} .
$$

For each $c>c_{0},\left(\frac{c}{c-w}, 0\right)$ is a saddle point of (4.1). The positive eigenvalue of the linearization is $\lambda_{1}=\frac{1}{c} r\left(\frac{c}{c-w}\right)$ and the corresponding eigenvector is $\phi_{1}=\left(-1 /\left[\left(\frac{c-w}{c}+\right.\right.\right.$ $\left.\left.\left.\frac{1}{c^{2}} r\left(\frac{c}{c-w}\right)\right)\right], 1\right)$. Clearly, this vector lies between the vectors $\left(-\frac{c}{c-w}, 1\right)$ and $(0,1)$. Therefore, in view of the stable manifold theorem, there exists a unique solution of problem (4.1) for $\eta<0$ that is tangential to $\phi_{1}$ at $\left(\frac{c}{c-w}, 0\right)$. Moreover, we show in Lemma 4.6 that the solution belongs to $\left\{(u, v): v+\frac{c-w}{c} u>1, u<\frac{c}{c-w}\right\}$.

In the analogous way to the case of $w>0$ we can consider the auxiliary problems (4.3)-(4.5). Lemmas 4.1 and 4.2 are still valid for $c>-w \frac{\theta}{1-\theta}$. The lower and upper solutions can be constructed as in (4.9) and (4.10). Then from Lemmas 4.1 and 4.2 we can infer the following estimates.

LEMma 4.6. Let $(u, v)$ be a solution of problem (4.6) for $c>-w \frac{\theta}{1-\theta}>0$, and let $(\underline{u}, \underline{v})$ and $(\bar{u}, \bar{v})$ be a pair of the lower and upper solutions of problems (4.7) and (4.8) for $(u, v)$ that are defined by (4.9) and (4.10). Then for $\eta \in(-\infty, 0]$

- $\theta \leq u(\eta)<\frac{c}{c-w}<1$ and $\frac{c-w}{c} u(\eta)+v(\eta)>1$,

- $\underline{v}(0)<v(0)<\bar{v}(0)$, and the trajectory of $(u(\eta), v(\eta))$ lies between two trajectories $(\underline{u}(\eta), \underline{v}(\eta))$ and $(\bar{u}(\eta), \bar{v}(\eta))$ for $\eta<0$, and

- $-c \bar{v}(0) \leq-c v(0) \leq u^{\prime}(\eta)<0$ and $0<v^{\prime}(\eta) \leq c r\left(\frac{c}{c-w}\right) v(0) \leq c r\left(\frac{c}{c-w}\right) \bar{v}(0)$.

Notice that for $w<0$ we cannot, in general, infer the monotonicity of $v(0)$ with respect to $c$ when $c>c_{0}$. In fact, we will show that the solutions are nonmonotonic in $c$, and solutions for different values of $c$ might intersect.

Figure 4.3 shows an illustration for Lemma 4.6. The heteroclinic orbits for different values of $c$ start from different saddle points for $\eta=-\infty$. The solution of problem (4.6) for $\eta<0$ is located between corresponding lower and upper solutions. Although 


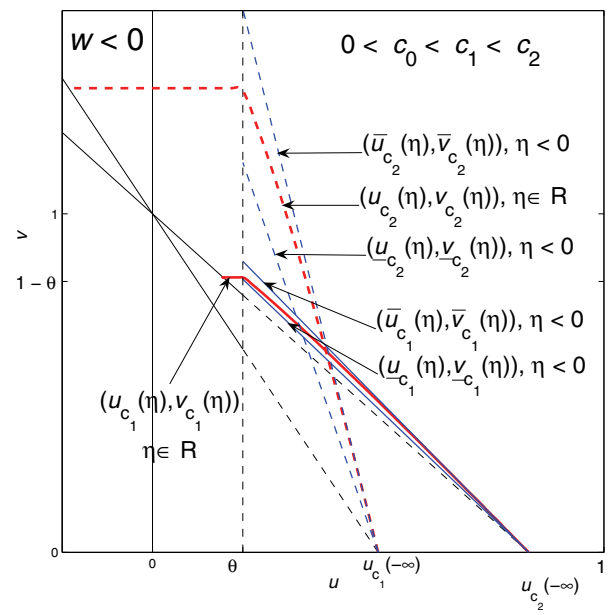

FIG. 4.3. Case $w<0$. Schematic phase plane of the $u$, $v$-system for two different positive values of $c$. The heteroclinic orbits $\left(u_{c_{1}}(\eta), v_{c_{1}}(\eta)\right)$ and $\left(u_{c_{2}}(\eta), v_{c_{2}}(\eta)\right), \eta \in \mathbb{R}$, connect the corresponding saddle stationary points distinct from $(1,0)$ with the corresponding set of stationary points. The values $\left(u_{c}(0), v_{c}(0)\right)$ do not vary monotonically with respect to $c$. The upper and lower solutions are shown for each heteroclinic orbit when $\eta<0$.

the solutions are nonmonotonic in $c$, we can still prove the existence of at least two wave speeds for $|w|$ small enough.

THEOREM 4.7. Let $w<0$.

(i) There exists a threshold $w_{*}<0$ given by (4.17) such that for each $w_{*}<w<0$ there exist at least two positive wave speeds $c_{1}$ and $c_{2}$, with $c_{1}<c_{2}$ such that for each of them the solution of problem (4.6) satisfies the conditions $v(0)=1$ and $u(0)=\theta$. Moreover,

$$
0<-w \frac{\theta}{1-\theta}<\bar{c}_{1}<c_{1}<\underline{c}_{1} \leq c_{*} \leq \underline{c}_{2}<c_{2}<\bar{c}_{2},
$$

where $c_{*}=\frac{1}{3}\left[2 w+\sqrt{w^{2}+3 \frac{1-\theta}{\theta} r(\theta)}\right], \bar{c}_{1}$ and $\bar{c}_{2}$ are two positive solutions of the equation $\bar{v}(0)=1$, and $\underline{c}_{1}$ and $\underline{c}_{2}$ are two positive solutions of the equation $\underline{v}(0)=1$.

(ii) There exists a threshold $w^{*}<0$ given by (4.18) such that for all $w<w^{*}<0$ there is no solution of problem (4.6) that satisfies the conditions $v(0)=1$ and $u(0)=\theta$.

Notice that $w^{*} \leq w_{*}$.

Proof. Based on the explicit solutions in (4.9) and (4.10) we can express $\underline{v}(0)$ and $\bar{v}(0)$ as functions of the wave speed $c$ :

$$
\begin{aligned}
& \underline{v}_{c}(0)=\left(1+\frac{1}{c(c-w)} r(\theta)\right)\left(1-\frac{c-w}{c} \theta\right), \\
& \bar{v}_{c}(0)=\left(1+\frac{1}{c(c-w)} r\left(\frac{c}{c-w}\right)\right)\left(1-\frac{c-w}{c} \theta\right) .
\end{aligned}
$$

It is clear that

$$
\lim _{c \rightarrow+\infty} \underline{v}_{c}(0)=\lim _{c \rightarrow+\infty} \bar{v}_{c}(0)=1-\theta, \quad \underline{v}_{c_{0}}(0)=\bar{v}_{c_{0}}(0)=0 .
$$

Thus, we study two cases. 


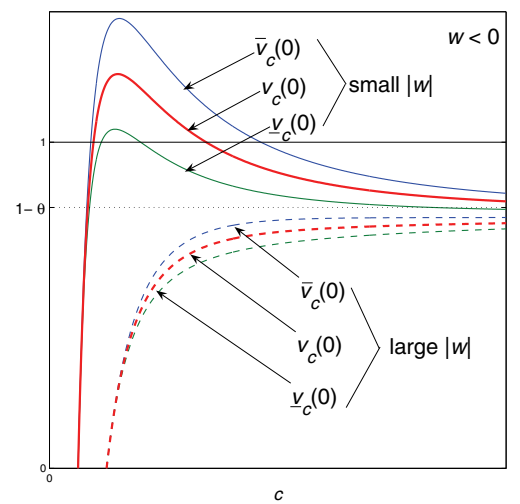

FIG. 4.4. Case $w<0$. Functions $v_{c}(0), \underline{v}_{c}(0)$, and $\bar{v}_{c}(0)$ for small and large values of $|w|$.

(i) If for the subsolution we have

$$
\underline{v}_{c}(0) \geq 1 \text { for some } c>c_{0},
$$

then $\bar{v}_{c}(0)>v_{c}(0)>\underline{v}_{c}(0) \geq 1$, and, therefore, there exist at least two values of $c$, say $c_{1}$ and $c_{2}$, such that $c_{0}<c_{1}<c_{2}$, and $v_{c_{1}}(0)=v_{c_{2}}(0)=1$.

(ii) On the other hand, if the supersolution satisfies

$$
\bar{v}_{c}(0) \leq 1 \quad \text { for all } \quad c>c_{0}
$$

then, in view of Lemma 4.6, there is no $c$ such that $v_{c}(0)=1$.

These two cases are illustrated in Figure 4.4.

Case (i). For $c>c_{0}$ the condition $\underline{v}_{c}(0) \geq 1$ is equivalent to $\underline{v}_{c}(0)-1 \geq 0$, which we write as

$$
\underline{f}(c, w)=\frac{c^{2}(c-w)\left(\underline{v}_{c}(0)-1\right)}{\theta}=-c^{3}+2 w c^{2}+c\left[\frac{1-\theta}{\theta} r(\theta)-w^{2}\right]+w r(\theta) \geq 0 .
$$

For this function we find that for $w<0$ we have $f\left(c_{0}, w\right)=-\frac{c_{0}^{2}\left(c_{0}-w\right)}{\theta}<0$, and $\lim _{c \rightarrow+\infty} \underline{f}(c, w)=-\infty$. Hence we check whether $f$ has a local maximum and whether this is positive for certain values of $c$ and $w$. For fixed $w<0$, the function $\underline{f}(c, w)$ attains its local maximum at

$$
c_{*}=\frac{2 w+\sqrt{w^{2}+3 \frac{1-\theta}{\theta} r(\theta)}}{3} .
$$

In order to be able to satisfy condition (4.15), we need to satisfy the two conditions (A) $c_{*}>c_{0}$ and $(\mathrm{B}) \underline{f}\left(c_{*}, w\right) \geq 0$ for appropriate wind values $w$. The inequality $c_{*}>c_{0}$ infers that

$$
-\sqrt{\frac{(1-\theta)^{3}}{(1+2 \theta) \theta} r(\theta)}=w_{0}<w<0
$$

To study the second condition (B), we observe that for $w=0$ we have $f\left(c_{*}, 0\right)>0$ and for $w=w_{0}$ we have $\underline{f}\left(c_{*}, w_{0}\right)<0$. Hence, there exists a value $w_{*}$ with $w_{0}<w_{*}<0$ such that 


$$
\underline{f}\left(c_{*}, w_{*}\right)=0 \quad \text { and } \quad \underline{f}\left(c_{*}, w\right)>0 \quad \text { for all } w_{*}<w<0 .
$$

Therefore, for each $w \in\left[w_{*}, 0\right)$, the inequality $\underline{v}_{c}(0) \geq 1$ (and equivalently $\underline{f}(c, w) \geq 0$ ) has a nonempty set of solutions $\left[\underline{c}_{1}, \underline{c}_{2}\right] \subset\left(c_{0}, \infty\right)$ with $c_{*} \in\left[\underline{c}_{1}, \underline{c}_{2}\right]$. In this case $c_{1}<\underline{c}_{1}$ and $c_{2}>\underline{c}_{2}$. The upper solution $\bar{v}$ can then be used to find further lower and upper bounds for the wave speeds. The bounds $\bar{c}_{1}$ and $\bar{c}_{2}$ are the solutions of the equation $\bar{v}_{c}(0)=1$ and they satisfy the inequalities $\bar{c}_{1}<c_{1}<\underline{c}_{1}$ and $\underline{c}_{2}<c_{2}<\bar{c}_{2}$; see also Figure 4.4.

Case (ii). The inequality (4.16) is certainly satisfied if

$$
\bar{v}_{c}(0)<\bar{v}_{c}(0)=\left(1-\frac{c-w}{c} \theta\right)\left(1+\frac{1}{c(c-w)} r(1)\right) \leq 1 .
$$

By analogy to Case (i), we can introduce the function $\overline{\bar{f}}(c, w)=\frac{c^{2}(c-w)\left(\overline{\bar{v}}_{c}(0)-1\right)}{\theta}$ and study conditions for $\overline{\bar{f}}(c, w) \leq 0$. We find that for fixed $w$ the interval $\left(c_{0}, \infty\right)$ does not contain the maximum of this function for

$$
w<w^{*}=-\sqrt{\frac{(1-\theta)^{3}}{(1+2 \theta) \theta} r(1) .}
$$

Since $\overline{\bar{f}}\left(c_{0}, w\right)=-\frac{c_{0}^{2}\left(c_{0}-w\right)}{\theta}<0$, the inequality $(4.16)$ is true for all $w<w^{*}$.

Now we can formulate the following result for the travelling wave problem in the case $w<0$.

TheOREM 4.8. Let $w<0$.

(i) There exists a threshold $w_{*}<0$ given by (4.17) such that for each $w_{*}<w<0$ system (2.1) has at least two travelling front solutions governed by equations (4.1). There are two different speeds $c_{1}$ and $c_{2}$ of travelling fronts that satisfy inequality (4.12). The trajectories of the travelling fronts $\left(u_{1}(\eta), v_{1}(\eta)\right)$ and $\left(u_{2}(\eta), v_{2}(\eta)\right), \eta \in \mathbb{R}$, corresponding to $c_{1}$ and $c_{2}$ belong to the respective domains

$$
\left\{(U, V): V+\frac{\underline{c}_{i}-w}{\underline{c}_{i}} U \geq 1,0 \leq V \leq 1,0 \leq U \leq \frac{\bar{c}_{i}}{\bar{c}_{i}-w}\right\}, \quad i=1,2 .
$$

Moreover,

$$
\begin{gathered}
0 \leq v_{i}^{\prime}(\eta) \leq \frac{1}{\underline{c}_{i}} r\left(\frac{\bar{c}_{i}}{\bar{c}_{i}-w}\right), \\
-\bar{c}_{i} \leq u_{i}^{\prime}(\eta)<0, \quad-r\left(\frac{\bar{c}_{i}}{\bar{c}_{i}-w}\right) \leq u^{\prime \prime}(\eta) \leq \bar{c}_{i}\left(\bar{c}_{i}-w\right), \quad i=1,2 .
\end{gathered}
$$

(ii) There exists a threshold $w^{*}<0$ given by (4.18) such that for all $w<w^{*}<0$ there are no travelling fronts for system (2.1) governed by equations (4.1).

Theorem 4.8 is illustrated in Figure 4.5(a) for small $|w|, w<0$. Figure 4.5(a) shows the existence of two solutions $\left(u_{1}(\eta), v_{1}(\eta)\right)$ and $\left(u_{2}(\eta), v_{2}(\eta)\right)$ that correspond to two different positive values for the speeds of the travelling wave $c_{1}$ and $c_{2}, 0<$ $c_{0}<c_{1}<c_{2}$. Figure 4.5(b) shows numerical approximations to the travelling fronts for $w=-0.1$ and $\theta=0.2$. In this case there are two solutions that correspond to the travelling fronts with the speeds $c_{1}=0.0378$ and $c_{2}=0.4037$. 


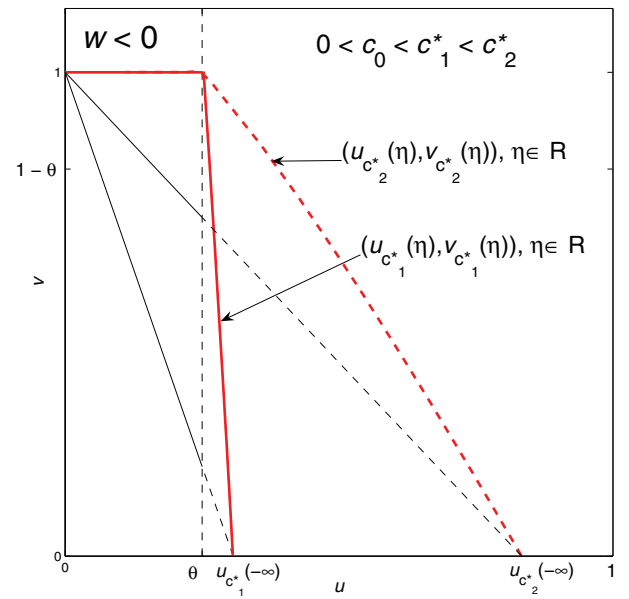

(a)

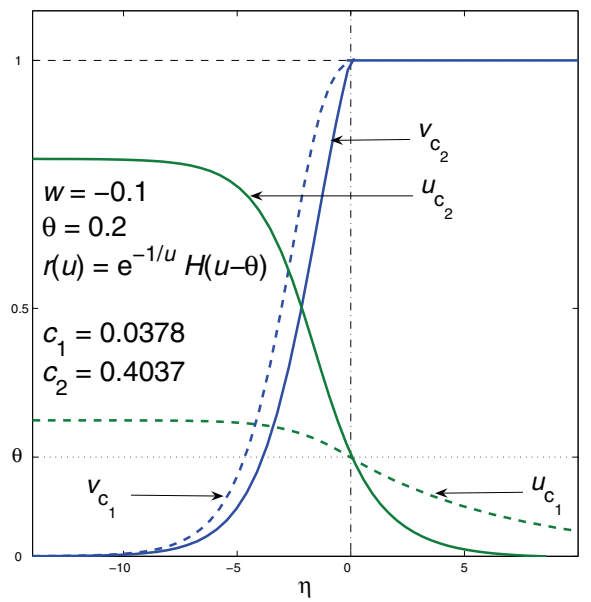

(b)

FIG. 4.5. Case $w<0$. (a) Schematic phase plane of the $u, v$-system for the solution of problem (4.1) with $w<0$. Two heteroclinic orbits $\left(u_{c_{1}}(\eta), v_{c_{1}}(\eta)\right)$ and $\left(u_{c_{2}}(\eta), v_{c_{2}}(\eta)\right), \eta \in \mathbb{R}$, connect the corresponding saddle stationary points $\left(c_{1} /\left(\left(c_{1}-w\right)\right), 0\right)$ and $\left(c_{2} /\left(\left(c_{2}-w\right)\right), 0\right)\left(c_{i} /\left(\left(c_{i}-w\right)\right)<1\right.$, $i=1,2)$ with the the point $(0,1)$ of the corresponding set of stationary points. (b) Numerical approximation to the travelling wave problem given by equations (4.1) for $w<0$. The figure shows two solutions to the problem for $w<0$.

\section{Case studies.}

5.1. Constant reaction rate. Let us first consider a constant reaction rate

$$
r(u)=r \mathcal{H}(u-\theta)
$$

in (2.1), where $\mathcal{H}$ denotes the Heaviside function. In this case the travelling wave speed $c=c(w)$ satisfies a simple algebraic equation

$$
\left(1+\frac{1}{c(c-w)} r\right)\left(1-\frac{c-w}{c} \theta\right)=1 .
$$

Figure 5.1(a) shows the relationship between $c$ and $w$. Since the mapping from $c \rightarrow w$ is uniquely defined for $c \in(0,+\infty)$, it is convenient to analyze the wind speed $w$ as a function of $c$ :

$$
w(c)=c-\frac{2 r c}{\theta r+\sqrt{(\theta r)^{2}+4 \theta r c^{2}}}=c-\frac{\sqrt{(\theta r)^{2}+4 c^{2} \theta r}-\theta r}{2 c \theta} .
$$

In such a case it is possible to predict how strong the wind should be to result in the fire front propagating with a given speed $c$.

From (5.2), the minimal value $w_{\min }$ of $w(c)$ is given by $w_{\min }=w\left(c_{\min }^{*}\right)<0$, where

$$
c_{\min }^{*}=\frac{1}{2} \sqrt{\frac{r}{2}(4-\theta-\sqrt{\theta(8+\theta)})} .
$$

The wind velocity $w_{\min }$ is a bifurcation point. If the wind velocity $w=w_{\min }$, the combustion problem given by (2.1) possesses a unique travelling wave speed $c_{\min }^{*}$. If the wind velocity $w$ belongs to the interval $\left(w_{\min }, 0\right)$, then the travelling front can 


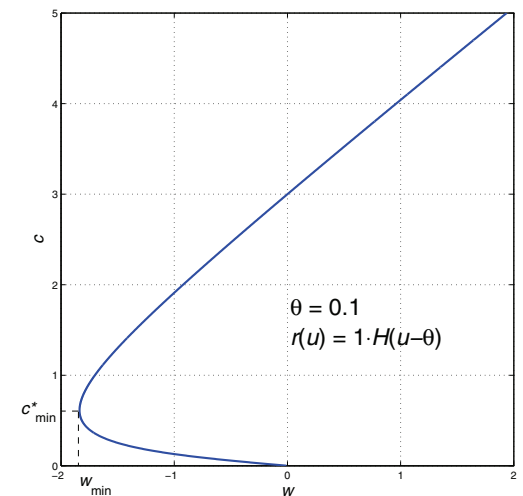

(a)

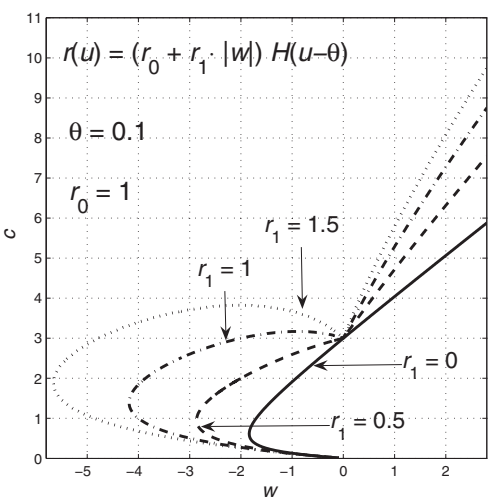

(b)

FIG. 5.1. (a) The relationship between the wind speed $w$ and the travelling front speed $c$ for a constant reaction term. (b) The relationship between the wind speed $w$ and the travelling front speed $c$ for a constant reaction term of the form $r_{0}+r_{1}|w|$. Here we show four values of $r_{1}=0,1,2,3$.

propagate with two different speeds $c_{1}<c_{\min }^{*}$ and $c_{2}>c_{\min }^{*}$. If the velocity is smaller than $w_{\min }$, then there are no solutions for the travelling wave problem. And in the case of nonnegative velocity there always exists a unique travelling wave speed $c$. Note also that in the case of constant reaction rate the critical thresholds $w^{*}$ and $w_{*}$ for nonexistence and nonuniqueness of travelling wave front (Theorem 4.8) are equal to $w_{\min }$.

Finally, compare the wind velocity and the travelling wave speed by considering the function $c-w$. It is easy to infer from (5.2) that $c-w$ is a strictly increasing and bounded function of $c$ for positive $c$; moreover,

$$
\lim _{c \rightarrow+0}(c-w)=0, \quad \lim _{c \rightarrow+\infty}(c-w)=\sqrt{\frac{r}{\theta}} .
$$

In the case of large wind towards unburned area, the difference between the corresponding travelling wave speed $c$ and the wind speed $w$ is almost constant. However, this difference varies significantly for small values of the wind speed.

5.2. Arrhenius reaction rate. A standard choice for the reaction rate is the Arrhenius law [26], i.e.,

$$
r(u)=e^{-\frac{\tilde{E}}{u+u_{\infty}}} \mathcal{H}(u-\theta),
$$

where $\tilde{E}$ and $u_{\infty}$ correspond to the rescaled activation energy and ambient temperature. In this case, the assumptions given in (2.2) are satisfied; therefore, for $\theta<1$ Theorems 3.6, 4.5, and 4.8 apply. We used the Arrhenius law in most of the illustrations in the earlier sections to illustrate the theoretical findings. In particular, Figures $3.3(\mathrm{~b}), 4.2(\mathrm{~b})$, and 4.5(b) illustrate the travelling wave solutions for the combustion problem (2.1) for $\theta=0.2$. The travelling wave speed for $w=0$ is approximated by $c=0.5682$ (Figure 3.3(b)). If the wind of speed $w=0.2$ blows in the direction of the moving fire (forward rate of spread), the travelling wave speed is $c=0.8370$ (Figure 4.2(b)). For an opposing wind (backward rate of spread) of speed $w=-0.2$ there are two travelling wave fronts: the slow front with the speed $c_{1}=0.0378$ and the fast front with the speed $c_{2}=0.4037$ (Figure 4.5(b)). Notice that in the case of the slow 
travelling wave speed $c_{1}$ the fuel is consumed much more slowly than in the case of fast speed $c_{2}$. Moreover, in the case of the slow travelling wave speed, the maximal temperature is very close to the ignition threshold $\theta$; therefore, the fire is of very low intensity.

5.3. Wind dependent reaction rate. In this section, we consider the case where the reaction rate $r(u,|w|)$ depends on the wind speed. For instance, the wind speed could result in an increase in the amount of oxygen available for the chemical reaction, or it could promote mixing through turbulence. In both those examples, the overall reaction rate would be increased when $|w|$ increases. This could be accounted for in the model by using the following modification to the reaction rate:

$$
r(u,|w|)=\left(r_{0}+r_{1}|w|\right) \mathcal{H}(u-\theta),
$$

where $r_{1}$ is a given constant. For fixed wind $w$, the above rate function fulfills all the assumptions (2.2); hence all of the above results apply. However, the threshold values, denoted by $c^{*}, w^{*}, w_{*}$, will now depend on $|w|$. The effect of the additional parameter $r_{1}$ on the travelling wave solution can be understood by rescaling the equations as follows. Define $\beta(|w|)=\frac{r_{0}+r_{1}|w|}{r_{0}}$ and rescale $t$ and $x$ according to $\tilde{t}=\beta t$ and $\tilde{x}=\sqrt{\beta} x$. In the rescaled coordinates, the system (2.1) becomes

$$
\begin{aligned}
& \frac{\partial u}{\partial t}+\frac{1}{\sqrt{\beta}} \vec{w} \cdot \tilde{\nabla} u=\tilde{\Delta} u+r_{0} \mathcal{H}(u-\theta) v, \\
& \frac{\partial v}{\partial t}=-r_{0} \mathcal{H}(u-\theta) v
\end{aligned}
$$

i.e., the solution for $r_{1} \neq 0$ and speed $|w|$ can be obtained from the solution for $r_{1}=0$ with rescaled speed $\frac{|w|}{\sqrt{\beta}}$. In particular, the speed of the travelling wave $c\left(r_{1}, w\right)$ can be simply obtained from the $r_{1}=0$ case using

$$
c\left(r_{1}, w\right)=c(0, w / \sqrt{\beta(|w|)}) \cdot \sqrt{\beta(|w|)} .
$$

This dependence of the wave speed $c$ on the wind $w$ is illustrated in Figure 5.1(b).

We observe that with $r_{1}>0$ the wind increases the speed $c$ of the fire front compared to the no-wind case. Moreover, we observe that if the perturbation term $r_{1}$ is large enough, then an opposing wind could possibly enhance combustion such that the backward rate of spread is increased as compared to no wind. Such effect has been reported in $[17,24]$ in conjunction with an increase in oxygen supply.

6. Stability analysis. The wind-wave speed diagram in Figure 5.1(a) immediately raises the question of the stability of the travelling wave solutions. We expect that for the negative wind speed the slower travelling wave is unstable. However, the full stability analysis is difficult, since it requires the analysis of a complex free boundary problem. Here we formulate only the corresponding free boundary problem and illustrate its solution with a few numerical examples. The complete analysis of the free boundary problem including existence and uniqueness of the free boundary problem and the stability of the travelling wave solutions will be deferred to another publication [1].

To avoid numerical difficulties that could arise for the problems with the moving boundary (at the unknown ignition front), we first transform the problem given by equations (2.1) to the problem attached to the ignition front. Assuming that $s(t)$ 
defines the location of ignition point at time $t$, i.e., $s(t)=\{\xi: u(t, \xi)=\theta\}$, we apply the change of variable in the form $y=x-s(t)$ to the system of equations (2.1) in one spatial dimension. Then $u(t, y)$ and $v(t, y)$ satisfy the following system of equations:

$$
\left\{\begin{array}{l}
u_{t}-\left(s^{\prime}(t)-w\right) u_{y}=u_{y y}+H(-y) r(u,|w|) v, \\
v_{t}-s^{\prime}(t) v_{y}=-H(-y) r(u,|w|) v
\end{array}\right.
$$

The numerical approximations for the system of equations (6.1) are obtained using a standard finite difference method on either side of the front, with an adapted grid that has a higher density of grid points in the vicinity of the ignition point $y=0$.

The speed of fire front $s^{\prime}(t)$ at time $t$ is computed using the equation

$$
\frac{d}{d t} \int_{-\infty}^{\infty} v(t, y) d y-\left.s^{\prime}(t) v\right|_{y=-\infty} ^{y=\infty}=-\int_{-\infty}^{0} r(u,|w|) v d y
$$

This equation is obtained by integrating the second equation of system (6.1) over the spatial domain $\mathbb{R}$.

The first derivative of the solution of (6.1) is discontinuous across the front (ignition point) $y=0$, and we need to pay special attention to how to match the numerical solutions on either side of the front. Based on the definition of the ignition point and the continuity condition for $u_{y}$ and $v$ we state the following matching conditions at $y=0$ :

$$
u(t, 0)=\theta, \quad\left[u_{y}(t, 0)\right]=0, \quad[v(t, 0)]=0 .
$$

Let $u_{2-}$ and $u_{-}$be the values of $u$ at the two nearest grid points to $y=0$ from the left-hand side and $u_{2+}$ and $u_{+}$from the right-hand side. Assuming that $u_{2-}$ and $u_{2+}$ were known, we defined $u_{-}$and $u_{+}$from the finite difference approximation of the second matching equation for $u$ and the equation

$$
\left[u_{y y}(t, 0)\right]=r(\theta+0,|w|) .
$$

The last equation was directly obtained from the first equation of system (6.1) and the matching conditions (6.2).

Here are some examples of numerical results. In our calculations we used a constant reaction rate given by (5.1) with $r=1$ and the ignition temperature $\theta=0.2$. Note that for the constant reaction rate the solutions of the travelling wave problem can be written in exact form.

The following observations were made: (1) in the case of zero wind and wind blowing from the burning region, the travelling wave solutions were stable; 2 ) in the case of wind blowing towards the burning region, the faster travelling waves were stable, and the slower travelling waves were always unstable. Even when the initial data were picked to coincide with the slower travelling wave solution, the numerical solution at later times always converged eventually to the corresponding faster travelling wave (upper branch in the diagram). Figure 6.1 shows the behavior of the fire front speed $s^{\prime}(t)$ for all types of wind velocity. The values of $s^{\prime}(t)$ converge to the travelling wave speeds for zero wind $w=0$ and the wind blowing towards the burning region (positive wind) $w=1>0$. In the case of the wind blowing towards the burning region (negative wind) $w=-0.5, s^{\prime}(t)$ converges to the larger travelling wave speed $c_{2}$ for small perturbations of both the faster and the slower travelling waves. 


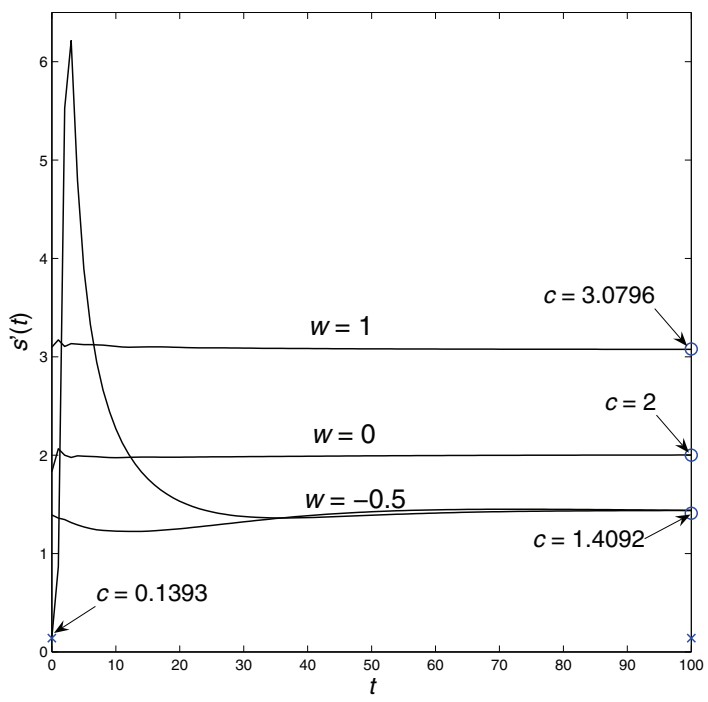

FIG. 6.1. Illustration of the behavior of the fire front speed for different wind velocity regimes.

7. Discussion. In this paper we use an adiabatic combustion model with a constant wind either in the direction of the moving fire front (forward rate of spread) or in the opposite direction (backward rate of spread). For the forward rate of spread we find that a wind of magnitude $w$ has an accelerating effect on the propagation speed compared to the case of $w=0$. To be more precise, we can show the following lemma.

LEMMA 7.1. The upper and lower bounds for the wave speeds $\underline{c}$ and $\bar{c}$ from Theorem 4.4 are monotonically increasing functions of the wind speed $w$.

Proof. The upper and lower bounds $\underline{c}, \bar{c}$ are determined by the conditions

$$
\underline{v}_{c}(0)=1 \quad \text { and } \quad \bar{v}_{c}(0)=1 \text {, }
$$

where $\underline{v}_{c}(0)$ and $\bar{v}_{c}(0)$ are given in (4.13) and (4.14), respectively. In the case of $w>0$ these functions are monotonically decreasing functions of $c$. Furthermore, we can study those expressions as functions of $w$, which we denote for now as $\underline{v}_{c}(0 ; w)$. Then for the subsolution we get

$$
\frac{d}{d w} \underline{v}_{c}(0 ; w)=\frac{1}{c(c-w)^{2}} r(\theta)+\frac{\theta}{c} \geq 0 .
$$

Hence $\underline{v}_{c}(0 ; w)$ is increasing as a function of $w$ and decreasing as a function of $c$. This means the point $c(w)$ given by the intersection $\underline{v}_{c}(0 ; w)=1$ satisfies

$$
\frac{d}{d w} c(w)=\frac{-d \underline{v} / d w}{d v / d c} \geq 0
$$

The upper bound $\bar{c}$ is also increasing in $w$. To see this we write $\bar{v}_{c}(0 ; w)=$ $H_{1}(w) H_{2}(w)$, with two increasing functions

$$
H_{1}(w)=1+\frac{1}{c(c-w)} r\left(\frac{c}{c-w}\right), \quad H_{2}(w)=1-\frac{c-w}{c} \theta .
$$


Then again $\bar{v}_{c}(0 ; w)$ is increasing in $w$ and decreasing in $c$; hence $\bar{c}(w)$ is increasing as a function of $c$.

LEMma 7.2. The travelling wave speed $c$ is a strictly monotonically increasing function of the wind speed $w$ for $w \geq 0$.

Proof. Assume, by contradiction, that $c(w)$ is not a strictly monotonically increasing function of $w$ for $w \geq 0$. Then, there exist two wind speeds $w_{1}$ and $w_{2}\left(0 \leq w_{1}<w_{2}\right)$ such that $c_{1}=c\left(w_{1}\right) \geq c_{2}=c\left(w_{2}\right)$. It is easy to see that in this case $u_{1}(-\infty)=\frac{c_{1}}{c_{1}-w_{1}}<$ $\frac{c_{2}}{c_{2}-w_{2}}=u_{2}(-\infty)$, where $\left(u_{i}(\eta), v_{i}(\eta)\right)=\left(u\left(\eta, w_{i}\right), v\left(\eta, w_{i}\right)\right), i=1,2$, for $\eta<0$.

Since $u_{1}(0)=u_{2}(0)=\theta$ and $v_{1}(0)=v_{2}(0)=1$, the two curves $\left(u_{1}(\eta), v_{1}(\eta)\right)$ and $\left(u_{2}(\eta), v_{2}(\eta)\right)$ need to have at least one intersection point for $\eta \leq 0$. Let $(\hat{u}, \hat{v})=$ $\left(u_{1}\left(\eta_{1}\right), v_{1}\left(\eta_{1}\right)\right)=\left(u_{2}\left(\eta_{2}\right), v_{2}\left(\eta_{2}\right)\right.$ be the closest intersection point of these curves to the line $v=0$. Then, using a geometric argument, the inequality $\frac{v_{1}^{\prime}\left(\eta_{1}\right)}{u_{1}^{\prime}\left(\eta_{1}\right)} \leq \frac{v_{2}^{\prime}\left(\eta_{2}\right)}{u_{2}^{\prime}\left(\eta_{2}\right)}$ holds. However, this leads to a contradiction, since

$$
\frac{r(\hat{u}) \hat{v}}{-c_{1}^{2}\left(\frac{c_{1}-w_{1}}{c_{1}} \hat{u}+\hat{v}-1\right)} \geq \frac{r(\hat{u}) \hat{v}}{-c_{2}^{2}\left(\frac{c_{1}-w_{1}}{c_{1}} \hat{u}+\hat{v}-1\right)}>\frac{r(\hat{u}) \hat{v}}{-c_{2}^{2}\left(\frac{c_{2}-w_{2}}{c_{2}} \hat{u}+\hat{v}-1\right)} .
$$

In the case of a wind opposing the fire spread direction (backward rate of spread), we find two qualitatively different cases. For large wind speed $|w|>\left|w^{*}\right|$ there is no self-similar solution in the form of a travelling wave. Notice that this does not exclude, however, backward spread of the fire. For small wind speed $|w|<\left|w_{*}\right|$ we find (at least) two travelling wave solutions. This is by far the most interesting case in our analysis. We strongly believe that there are indeed exactly two possible solutions. For the sake of the following arguments we call them the slow and the fast wave. As seen from the profile in Figure 4.5(b), the fast wave shows a large temperature and it looks similar to the forward wave in Figure 4.2(b). To our understanding, this represents the fully developed fire that burns on a self-sustained temperature. The slow wave, seen in Figure 4.5(b), has a burning temperature just above threshold. This solution has features that resemble those of a smoldering wave. Here the temperature is slightly above the ignition temperature, but the fire has not started to burn. Heat is transported very slowly through smoldering [4]. In nature, the smoldering fires may persist for quite a long time. However, the numerical experiments of our idealized model show the instability of existing slow combustion waves. Here we would like to note that our model is an idealized model for forest fire spread. It gives us quite a realistic description of forest fires but at the same time neglects many physical processes in combustion. We believe that the inclusion of more detailed physical factors in the model would lead to a better description of the nature of the slow travelling waves, in particular to their stability. Currently, we are performing a systematic linear stability analysis to investigate the stability of travelling wave solutions [1].

One important assumption of this article is the absence of heat loss. We have also started a similar study for a model with heat loss. Preliminary numerical simulations show that, as before, and despite the inclusion of a heat loss term, we obtain two travelling waves for small negative and small positive wind velocities [2]. This confirms our belief that the slow wave has a physical meaning. This needs, however, to be investigated further.

Finally, we included the wind speed in the reaction rate. We showed that an increased combustion rate due to increased oxygen supply can significantly increase forward and backward fire front speed. A more detailed modelling of oxygen dynamics needs to follow. 
Acknowledgments. We thank Cordy Tymstra (Alberta Sustainable Resource and Development) and the members of the Forest Fire Discussion Group at the University of Alberta for valuable suggestions. This work is part of the MITACS project on Forest Fires and Spread in Heterogeneous Landscapes.

\section{REFERENCES}

[1] P. Babak, A. Bourlioux, and T. Hillen, Stability of Wind Driven Fire Fronts for an Idealized Forest Fire Model, manuscript, 2009.

[2] P. Babak, A. Bourlioux, and T. Hillen, Wind Driven Fire Fronts for an Idealized Forest Fire Model with Heat Loss, manuscript, 2009.

[3] A. BAyliss And B. J. MatKowsky, Two routes to chaos in condensed phase combustion, SIAM J. Appl. Math., 50 (1990), pp. 437-459.

[4] E. A. Johnson And K. Miyanishi, Eds., Forest Fires: Behavior and Ecological Effects, Academic Press, San Diego, 2001.

[5] E. Logak And V. Loubeau, Travelling wave solutions to a condensed phase combustion model, Asymptot. Anal., 12 (1996), pp. 259-294.

[6] E. LOGAK, Mathematical analysis of a condensed phase combustion model without ignition temperature, Nonlinear Anal., 28 (1997), pp. 1-38.

[7] E. I. Maksimov and A. G. Merzhanov, Theory of combustion of condensed substances, Combustion, Explosion and Shock Waves, 2 (1966), pp. 25-31.

[8] J. Mandel, L. S. Bennethum, J. D. Beezley, J. L. Coen, C. C. Douglas, M. Kim, and A. Vodacek, A wildland fire model with data assimilation, Math. Comput. Simulation, 79 (2008), pp. 584-606.

[9] S. B. MARGOLIS, An asymptotic theory of condensed two-phase flame propagation, SIAM J. Appl. Math., 43 (1983), pp. 351-369.

[10] G. N. Mercer, R. O. Weber, And H. S. Sidhu, An oscillatory route to extinction for solid fuel combustion waves due to heat losses, R. Soc. Lond. Proc. Ser. A Math. Phys. Eng. Sci., 454 (1998), pp. 2015-2022.

[11] F. Morandini, P. A. Santoni, J. H. Balbi, J. M. Ventura, and J. M. Mandes-Lopes, A two-dimensional model of fire spread across a fuel bed including wind combined with slope conditions, Int. J. Wildland Fire, 11 (2002), pp. 53-64.

[12] F. Morandini, A. Simeoni, P. A. Santoni, and J. H. Balbi, A model for the spread of fire across a fuel bed incorporating the effects of wind and slope, Combust. Sci. Technol., 177 (2005), pp. 1381-1418.

[13] J. H. PARK, A. Bayliss, and B. J. Matkowsky, The transition from spinning to radial solid flame waves, Appl. Math. Lett., 17 (2004), pp. 123-131.

[14] J. H. Park, A. Bayliss, and B. J. Matkowsky, Dynamics in a rod model of solid flame waves, SIAM J. Appl. Math., 65 (2005), pp. 521-549.

[15] C. P. Please, F. Liu, And D. L. S. McElwain, Condensed phase combustion travelling waves with sequential exothermic or endothermic reactions, Combust. Theory Model., 7 (2003), pp. 129-143.

[16] B. Porterie, D. Morvan, J. C. Loraud, and M. Larini, Firespread through fuel beds: Modeling of wind-aided fires and induced hydrodynamics, Phys. Fluids, 12 (2000), pp. 1762-1782.

[17] J. S. RoH, S. S. YANG, AND H. S. RYOU, Tunnel fires: Experiments on critical velocity and burning rate in pool fire during longitudinal ventilation, J. Fire Sci., 25 (2007), pp. 161-175.

[18] F. J. Seron, D. Gutierrez, J. Magallon, L. Ferragut, and M. I. Asensio, The evolution of a WILDLAND forest FIRE FRONT, The Visual Computer, 21 (2005), pp. 152-169.

[19] K. G. Shkadinsky, B. I. Khaikin, and A. G. Merzhanov, Propagation of a pulsating exothermic reaction front in the condensed phase, Combustion, Explosion and Shock Waves, 7 (1971), pp. 15-22.

[20] A. Simeoni, P. A. Santoni, M. Larini, And J. H.Balbi, On the wind advection influence on the fire spread across a fuel bed: Modelling by a semi-physical approach and testing with experiments, Fire Saf. J., 36 (2001), pp. 591-513.

[21] D. V. Viegas, Slope and wind effects on fire propagation, Int. J. Wildland Fire, 13 (2004), pp. $143-156$.

[22] V. A. Volpert And V. A. Volpert, Propagation velocity estimation for condensed phase combustion, SIAM J. Appl. Math., 51 (1991), pp. 1074-1089.

[23] I. Volpert, V. Volpert, and V. Volpert, Traveling Wave Solutions of Parabolic Systems, Transl. Math. Monogr. 140, AMS, Providence, RI, 1994. 
[24] H. Y. WANG AND P. Joulain, Numerical simulation of wind-aided flame propagation over horizontal surface of liquid fuel in a model tunnel, J. Loss Prev. Process Ind., 20 (2007), pp. 541-550.

[25] R. O. Weber, G. N. Mercer, H. S. Sidhu, and B. F. Gray, Combustion waves for gases $(L e=1)$ and solids $(L e \rightarrow \infty)$, Proc. Roy. Soc. London Ser. A, 453 (1997), pp. 1105-1118.

[26] F. A. Williams, Combustion Theory, Addison-Wesley, New York, 1985. 